\title{
Prevalence of High Intensity Zone in LumbarSpine and its Correlation with Lumbar Disc Degeneration in Patients Attending Tertiary Hospital of Eastern Nepal
}

\section{Subash Chandra Jha, ${ }^{1}$ Satendra Raut, ${ }^{2}$ Pradip Kumar Gupta, ${ }^{1}$ Surya Parajuli ${ }^{3}$}

1Department of orthopedics, 2Department of Radiology, 3Department of Community Medicine, Birat Medical College and Teaching Hospital, Biratnagar, Morang, Nepal.

\section{ABSTRACT}

\section{Introduction}

A high intensity zone (HIZ) in intervertebral disc (IVD) of lumbar spine is a high intensity signal located in the annulus fibrosus of T2-weighted magnetic resonance (MR) imaging. There is limited information on the prevalence of HIZ in lumbar spine in Nepalese population. The aim of this cross-sectional study was to identify the prevalence of HIZ according to the age and its correlation with degenerated disc, disc herniation \& bulge and modic changes.

\section{Methods}

This was a prospective MR based cross-sectional study in 268 patients (1380 IVD) with LBP, leg pain or numbness. Data was collected from the hospital database of radiology department where single blinding was maintained and was analysed using SPSS version 23.

\section{Results}

The prevalence of HIZ in patients with LBP was 30.5\%. Degenerated disc was observed in $95 \%(116 / 122)$, disc herniation \& bulge in $98.3 \%(120 / 122)$ and Modic changes in $11.4 \%$ $(14 / 122)$ of the discs with HIZ.

\section{Conclusion}

There was statistically significant correlation between existence of HIZ and degenerated disc and disc herniation \& bulge, but there was no correlation with the existence of Modic changes.

Keywords: disc degeneration; HIZ; low back pain.

Correspondance: Subash Chandra Jha, Department of Orthopedics, Birat Medical College and Teaching Hospital, Nepal. Email: subash.jha@gmail.com, Phone: +977-9803012971. 


\section{INTRODUCTION}

A high intensity zone (HIZ) represents the detached nucleus pulposus which is trapped between the lamellae of torn annulus fibrosus, which causes a characteristic high signal change in magnetic resonance (MR) imaging , following inflammation. ${ }^{1-3}$ The term HIZ was first described by April and Bogdok in 1992 with prevalence of $28 \%$ in symptomatic individual. ${ }^{1}$ Prevalence of HIZ varies form $28-59 \%$ in symptomatic individuals but its identification in asymptomatic individuals has questioned its significance in clinical practice. ${ }^{4-10}$

To increase the diagnostic accuracy of HIZ, many authors now suggests to differentiate true HIZ from low/medium intensity zone depending on signal intensity on MR imaging. ${ }^{3,}$ 5-8 The purposes of this study were to investigate: (i) the prevalence of HIZ in patients with LBP in different age groups and (ii) the correlation between HIZ and other degenerative findings on lumbar MR images (like disc degeneration, disc bulge \& herniation and adjacent Modic changes).

\section{METHODS}

This was a prospective cross-sectional study. A total of 268 (n) consecutive patients were included from the hospital database of radiology department. All those patients who underwent MR imaging of the lumbar spine for symptoms like LBP, leg pain and numbness, between January 2020 to November 2020 were included in the study. Patients with infective spondylitis, tumor and who had history of surgery of lumbar spine were excluded. The study protocol was approved by the institutional review committee at Birat Medical College and Teaching Hospital

While collecting the data from the hospital database, single blinding was maintained. All the information required for the study was recorded in a separate paper for each participant and was double entered in the personal computer.
All patients were divided into 5 age groups $<20$ years, 20-39 years, 40-59 years, 60-79 years, $>80$ years. Prevalence of HIZ was calculated according to the above mentioned age groups. A 1.5 tesla MRI scanner (Signa Explorer, GE healthcare, Chicago, Illinois, USA) was used to acquire images, T1 weighted (335<repetition time $<400 \mathrm{~m} / \mathrm{sec}$; echo time $=42 \mathrm{~m} / \mathrm{sec})$ and $\mathrm{T} 2$ weighted $(3000<$ repetition time $<5000 \mathrm{~m} / \mathrm{sec}$; echo time $=120 \mathrm{~m} / \mathrm{sec}$ ) spine echo images were taken in the sagittal, coronal and axial planes. MR images had a slice thickness of $4 \mathrm{~mm}$ except axial were $3 \mathrm{~mm}$.

HIZ was defined as a bright white signal located in the substance of the posterior or posterolateral annulus fibrosus, that was clearly dissociated from the nucleus pulposus and surrounded by the low intensity (black) signal of annulus fibrosus, and was appreciably brighter than that of the nucleus pulposus in T2-weighted sequence on MR images. ${ }^{1-3}$ We differentiated true HIZ from low/medium intensity zone by presence of significantly high intensity signal which was as intense as cerebro-spinal fluid (CSF) signal located adjacent to the disc. (Figure 1). Along with that we also analysed statistical correlation between (i) HIZ and degenerated disc, (ii) HIZ and disc bulge \& herniation (iii) $\mathrm{HIZ}$ and change in adjacent endplates i.e. Modic changes.
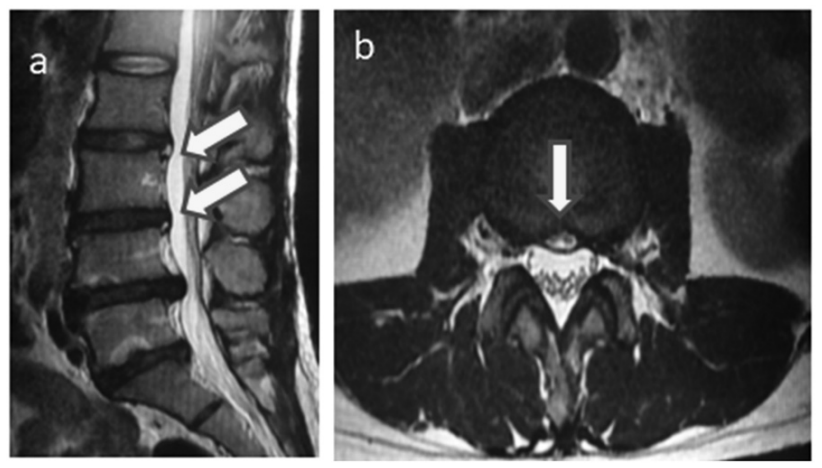

Figure 1. (a) Sagittal T2-weighted magnetic resonance image showing a high-intensity zone (arrows) within the posterior annulus at L2-3 and L3-4 (b) Axial T2-weighted magnetic resonance image showing a high intensity zone (arrow) within the posterior annulus at L3-4. 
Disc degeneration was assessed on T2weighted image on the basis of Pfirmann grading system ${ }^{11}$ as follows: grade 1: normal structure, no horizontal bands, clear distinction between annulus and nucleus; grade 2: inhomogeneous structurewith horizontal bands , clear distinction between nucleus and annulus; grade 3: inhomogeneous structure with unclear distinction between nucleus and annulus, features of annulus still recognizable; grade 4: inhomogeneous structure with hypointensity, shape of annulus not intact and no distinction between nucleus and annulus, disc height usually decreased and grade 5: same as grade 4 using Chi-square test. For all of the statistical test $p<0.05$ was considered statistically significant. Statistical analysis were performed using statistical package for social sciences (SPSS version 23.0 for window, SPSS. Inc. Chicago.IL).

\section{RESULTS}

Total 268 patients with low back pain were included in the study with 126 males and 142 females. The mean age of patient was 48.18years (16-88 years). Eighty two (30.5\%) patients had HIZ in at least one level of IVD. Most of the HIZ (93.4\%) were observed in age group of 20-59 years (Table 1). Total 1340 lumbar intervertebral disc

Table 1. Distribution of patients according to the age group and levels involved with $\mathrm{HIZ}$.

\begin{tabular}{|l|l|l|l|l|}
\hline Lumbar IVD & HIZ (\%) & Degenerated disc (\%) & Disc herniation \& bulge (\%) & Modic change (\%) \\
\hline L1-2 & $2(3.2)$ & $84(11.3)$ & $50(7.2)$ & $10(5.4)$ \\
\hline L2-3 & $22(18)$ & $116(15.6)$ & $92(13.3)$ & $16(8.7)$ \\
\hline L3-4 & $24(19.6)$ & $140(18.8)$ & $128(18.6)$ & $34(18.6)$ \\
\hline L4-5 & $54(44.2)$ & $204(27.4)$ & $236(34.3)$ & $72(39.5)$ \\
\hline L5-S1 & $18(14.7)$ & $198(26.6)$ & $182(26.4)$ & $50(27.4)$ \\
\hline Total & 120 & 742 & 688 & 182 \\
\hline
\end{tabular}

IVD: Intervertebral Disc, HIZ: High Intensity Zone

but disc space collapsed. For evaluation of data, Pfirmann grade 1-3 were categorized as normal and grade $4-5$ as degenerated disc.

Disc herniation \& bulge were classified by Fardon et al. ${ }^{12}$, where herniation was defined as disc displacement of disc material involving less than $50 \%$ of the disc circumference, and bulge was defined as disc displacement involving $>50 \%$ of the disc circumference. Vertebral endplate and bone marrow changes were graded as absent, type 1 (hypointense in T1-weighted sequence and hyperintense in T2-weighted sequence), type 2 (hyperintense in both sequence) and type 3 (hypointense in both sequence) as defined by Modic et al. ${ }^{13}$ Difference of ratio were compared
(IVD) were evaluated for HIZ, disc degeneration, disc herniation \& bulge and Modic changes. L4-5 IVD had maximum number of HIZ along with disc degeneration, disc herniation \& bulge and Modic changes (Table 2).

Disc degeneration was observed in 95\% $(116 / 122)$ and $51.3 \%(626 / 1218)$ of disc with and without HIZ respectively. The prevalence of degeneration in disc with HIZ was significantly higher than that in disc without HIZ (Table 3). Disc herniation \& bulge were observed in $98.3 \%$ (120/122) and $46.6 \%$ (568/1218) of the disc with and without HIZ, respectively. Prevalence of herniation \& bulge in disc with HIZ was significantly higher than that in disc without 
HIZ (Table 4). Modic changes were observed in $11.4 \%(14 / 122)$ and $13.7 \%(168 / 1218)$ of disc with and without HIZ respectively. No statistically significant difference was observed between the two groups (Table 5).

\begin{tabular}{|c|c|c|c|c|c|c|}
\hline $\begin{array}{c}\text { Age group } \\
\text { (years) }\end{array}$ & $0-19$ & $20-39$ & $40-59$ & $60-79$ & $>80$ & Total \\
\hline HIZ in 3 IVDs & 0 & 0 & 6 & 0 & 0 & 6 \\
\hline HIZ in 2 IVDs & 0 & 10 & 18 & 0 & 0 & 28 \\
\hline HIZ in 1 IVD & 0 & 18 & 22 & 8 & 0 & 48 \\
\hline HIZ absent & 6 & 52 & 74 & 50 & 4 & 186 \\
\hline Total & 6 & 80 & 120 & 58 & 4 & 268 \\
\hline
\end{tabular}

HIZ: High Intensity Zone; IVD: Inter-Vertebral Disc

Table 2. Distribution of HIZ, Degenerated disc, disc herniation \& bulge and Modic changes at each lumbar IVD.

\begin{tabular}{|l|r|r|r|r|}
\hline \multirow{2}{*}{ Normal } & \multicolumn{2}{c|}{ Disc degeneration } & \multirow{2}{*}{ Total } \\
\cline { 3 - 4 } & Degenerated & & \\
\hline \multirow{3}{*}{ HIZ } & Present & 6 & 116 & 122 \\
\cline { 2 - 5 } & Absent & 592 & 626 & 1218 \\
\hline Total & 598 & 742 & 1340 \\
\hline
\end{tabular}

HIZ: High Intensity Zone

Table 3. Correlation between Disc degeneration and HIZ. $P$ value $=0.000$

\begin{tabular}{|l|c|c|c|c|}
\hline & \multicolumn{3}{|c|}{ Disc herniation \& bulge } & \multirow{2}{*}{ Total } \\
\hline \multirow{2}{*}{ HIZ } & & Present & Absent & \\
\cline { 2 - 5 } & Present & 120 & 2 & 122 \\
\hline Total & & 688 & 652 & 1340 \\
\hline
\end{tabular}

HIZ: High Intensity Zone

Table 4: Correlation between Disc herniation \& bulge and HIZ. $P$ value $=0.000$.

\begin{tabular}{|c|c|c|c|c|c|c|}
\hline & \multicolumn{4}{|c|}{ Modic changes } & \multirow{3}{*}{ Total } \\
\hline & & \multirow{2}{*}{ Absent } & \multicolumn{3}{|c|}{ Present } & \\
\hline & & & Type 1 & Type 2 & Type 3 & \\
\hline \multirow{2}{*}{ HIZ } & Present & 108 & 2 & 12 & 0 & 122 \\
\hline & Absent & 1050 & 12 & 152 & 4 & 1218 \\
\hline \multicolumn{2}{|c|}{ Total } & 1158 & 14 & 164 & 4 & 1340 \\
\hline
\end{tabular}

HIZ: High Intensity Zone
Table 5: Correlation between Modic change and HIZ. P value $=0.669$.

\section{DISCUSSION}

The presence of granulation tissue or neovascularisation induced by inflammation was first demonstrated by Ross et al. ${ }^{14}$ Such inflammatory granulation tissue produces pro-inflammatory cytokines and mediators which sensitize the neuroreceptors within the disc causing pain. These findings suggest that biomechanical mediators are more important than mechanical compression in the pathogenesis of DLBP. ${ }^{15,16}$ Low/medium intensity zone can represent annular fissure in asymptomatic population. ${ }^{17,} 18$ In normal adult, the annulus fibrosus is innervated by the recurrent meningeal nerve and by branches from the ventral ramus of the somatic spinal nerve. Most of the nerve supply of the IVD is limited to the periphery of the annulus fibrosus. ${ }^{1,}$ Renkine et al. ${ }^{5}$ reported that maximum number of HIZ are present over posterior annulus followed by posterolateral annulus. It is because posterior annulus fibrosus is structurally weak and exposed to high stress concentration. ${ }^{20}$

Bugdok $^{18}$ and Liu et al. ${ }^{3}$ emphasized that HIZ must be an intense signal rather than any bright spot on T2weighted MR image. Schellhas et al. ${ }^{21}$ reported that $87 \%$ of the HIZ positive discs were concordantly painful on discography while 65 of $67 \mathrm{HIZ}$ negative disc didn't elicit concordant pain on discography. Hence, concluded that the presence of HIZ was sign of internal disc disruption.

Takeuchi et al. ${ }^{10}$ evaluated prevalence of HIZ by age and correlation of HIZ with disc degeneration, disc bulge \& herniation and modic changes. They concluded that there was no significant change in the prevalence of HIZ beyond the age of 20 years, although they have included only small number of patients aged 
less than 20 years. They demonstrated close correlation of HIZ with disc degeneration, disc bulge \& herniation in patients with LBP, leg pain or numbness. They anticipated that the diagnostic value of HIZ may be higher in patients under age of 20 years, but they had only two patients who had symptomatic HIZ in this age group. Jha et $\mathrm{al}^{7}$ identified that the presence of HIZ even in one IVD is directly proportional to increased incidence of disc degeneration in remaining lumbar IVD, mainly in the lower three lumbar discs. They predicted that HIZ positive patients are more susceptible for degeneration of adjacent level disc compared to HIZ negative patients. In their study of $43 \mathrm{HIZ}$ positive patients out of 288 patients, 34 had single level HIZ positive disc and nine had multilevel HIZ with two levels in eight patients and three levels in one patient. They also demonstrated $87 \%$ had $\mathrm{HIZ}$ at posterior annulus and $13 \%$ had HIZ at postero-lateral annulus. Similarly, Lam et al ${ }^{22}$ demonstrated significant correlation between abnormal disc disruption and presence of HIZ .

This study had few limitations, like we included

\section{REFERENCES}

1. Aprill C, Bogduk N. High intensity zone: a diagnostic sign of painful lumbar disc on magnetic resonance imaging. Br. J. Radiol. ; 65 (773): 361-9. 1992. DOI: 10.1259/0007-1285-65-773-361

2. Jha SC, Higashino K, Sakai T, Takata Y, Abe M, Yamashita K, et al. Clinical significance of high intensity zone for discogenic low back pain: A review. J Med Invest; 63 (1-2):1-7, 2016. DOI: 10.2152/jmi.63.1.

3. Liu C, Cai H-X, Zhang J-F, Ma J-J, Lu Y-J, Fan S-W: Quantitative estimation of the high-intensity zone in the lumbar spine: a very small number of patients representing age group less than 20 or more than 80 years which may contribute to the bias in statistics. Therefore a bigger sample population will be required in future studies. Secondly, it is difficult to say that the HIZ was the cause for LBP in these patients because LBP can be due to various other reasons such as osteoarthritis of facet joint, spinal deformities or the muscular cause etc.

\section{CONCLUSIONS}

Prevalence of HIZ in patients with LBP in Nepal is comparable to the literature worldwide. There is statistically significant relation between (i) HIZ and degenerated disc (ii)HIZ and disc bulge \& herniation. More than $90 \%$ of HIZ were present in active age group of 20-59. Prevalence of HIZ, degenerated disc, disc herniation \& bulge and Modic changes were mostly in the lower three lumbar IVD.

\section{ACKNOWLEDGEMENTS}

We would like to express our great appreciation to Institutional review committee of Birat Medical College and Teaching Hospital.

comparison between the symptomatic and asymptomatic population. Spine J; 391-396, 2014. DOI: 10.1016/j. spinee.2013.06.078

4. Carragee EJ, Paragioudakis SJ, Kharana S: 2000 Volvo award winner in clinical studies: Lumbar high-intensity zone and discography in subjects without low back pain problems. Spine 25; 2987-2992, 2000. DOI: 10.1097/00007632-20001201000005

5. Rankine JJ, Gill KP, Hutchinton CE, Ross ER, Williamson JB: The clinical significance of the high-intensity zone on lumbar spine magnetic resonance 
imaging. Spine 24; 1913-1920, 1999. DOI: 10.1097/00007632-199909150-00009

6. Mitra D, Cassar-Pullicino VN, McCall IW. Longitudinal study of high intensity zones on MR of lumbar intervertbral discs, ClinRadiol 59: 1002-1008, 2004. DOI: 10.1016/j.crad.2004.06.001

7. Jha SC, Takata Y, Abe M, Yamashita K, Tezuka F, Sakai T, et al. High intensity zone in lumbar spine and its correlation with disc degeneration. J Med Invest. 2017;64(1.2):39-42. doi: 10.2152/jmi.64.39

8. Ito M, Incorvaia KM, Yu SF, Fredrickson BE, Yuan HA, Rosenbaum AE: Predictive signs of discogenic lumbar pain on magnetic resonance imaging with discography correlation. Spine 23: 1252-1260, 1998 DOI: 10.1097/00007632199806010-00016

9. O'Neill C, Kurgansky M, Kaiser J, Lau W: Accuracy of MRI for diagnosis of discogenic pain. Pain Physician 11: 311326, 2008.

10. Takeuchi M, Nagamachi A, Adachi $\mathrm{K}$, Inoue $\mathrm{K}$, Tamaki $\mathrm{Y}$, Omichi $\mathrm{Y}$, et al. Prevaence of High intensity zone in the lumbar spine according to age and their correlation with other degenerative findings on magnetic resonance imaging. Spine SurgRelat Res; 2 (4): 299-303, 2018. DOI: $10.22603 /$ ssrr.2017-0071

11. Pfirrmann CW, Metzdorf A, Zanetti M, J Hodler, N Boos. Magnetic resonance classification of lumbar intervertebral disc degeneration. Spine; 26(17): 1873-8, 2001. DOI: 10.1097/00007632-20010901000011

12. Fardon DF, Milette PC. The combined task forces of the NorthAmericanSpineSociety,
American Society of Sine Radiology, and American Society of Neuroradiology nomenclature and classification of lumbar disc pathology. Spine; 26(5): E93-113, 2001. DOI: 10.1097/00007632-200103010-00006

13. Modic M, Steinberg P, Ross J, Masaryk $\mathrm{T}$, Carter J. Degenerative disc disease: assessment of changes in vertebral body marrow in MR imaging. Radiology; 166(1): 193-9, $1988 . \quad$ DOI: 10.1148/ radiology.166.1.3336678

14. Ross JS, Modic MT, Masaryk TJ. Tears of the annulus fibrosus: assessment with GdDTPA enhanced MR imaging. AJR Am. J Roentgenol: 154: 159-162, 1990.

15. Burke JG, Watson RW, McCormack D, Dowling FE, Walsh MG, Fitzpatrick JM: Intervertebral disc which cause low back pain secretehigh levels of proinflammatory mediators. J Bone Joint Surg Br. 4: 196-201, 2002

16. Kaiser JA; Point of view. Lumbar dischighintensity zone Correlation of magnetic resonance imaging and discography. Spine 21: 86, 1996

17. Liu C, Cai HX, Zhang JF, Ma JJ, Lu YJ, Fan SW. Quantitative estimation of the high-intensity zone in the lumbar spine: comparison between the symptomatic and asymptomatic population. Spine J 14(3): 391-396, 2014. DOI: 10.1016/j. spinee.2013.06.078

18. Bogduk N. Point of view. Predictive signs of discogenic lumbar pain on magnetic resonance imaging with discography correlation. Spine 23: 1259-60, 1998.

19. Bogduk N, Tynan W, Wilson AS. The nerve supply of the human lumbar intervertebral disc. J Anat 132: 39-56, 1981 
20. Wang ZX, Hu YG: Clinical investigation of high intensity zone in anterior annulus fibrosus of lumbar disc; compared with high-intensity zone in posterior annulus fibrosus. ZhonghuaWaiKeZaZhi 47: 689692, 2009 PMID: 19615240

21. Schellhas KP, Pollei SR, Gundry CR, Heithoff KB. Lumbar disc high intensity zone.Correlation of magnetic resonance imaging and discography. Spine 21: 79-86, 1996 DOI: 10.1097/00007632-19960101000018

22. Lam KS, Carlin D, Mulholland RC. Lumbar disc high-intensity zone: the value and significance of provocative discography in the determination of the discogenic pain source. Eur Spine J; 9 (1): 36-41. 2000. DOI: $10.1007 / \mathrm{s} 005860050006$

Citation: Jha S, Raut S, Gupta P, Parajuli S. Prevalence of High Intensity Zone in the Lumbar Spine and its Correlation with Lumbar Disc Degeneration in Patients Attending Birat Medical College and Teaching Hospital. JCMS Nepal. 2021; $17(1) ; 81-7$. 